Research Article

\title{
Optimum Location of Autonomous Vehicle Lanes: A Model Considering Capacity Variation
}

\author{
Sara Movaghar, ${ }^{1}$ Mahmoud Mesbah ${ }^{D},{ }^{1,2}$ and Meeghat Habibian ${ }^{1}$ \\ ${ }^{1}$ Amirkabir University of Technology (Tehran Polytechnic), Tehran, Iran \\ ${ }^{2}$ The University of Queensland, Brisbane, Australia \\ Correspondence should be addressed to Mahmoud Mesbah; mmesbah@aut.ac.ir
}

Received 24 December 2019; Revised 26 February 2020; Accepted 18 March 2020; Published 12 May 2020

Academic Editor: Anna M. Gil-Lafuente

Copyright ( $(2020$ Sara Movaghar et al. This is an open access article distributed under the Creative Commons Attribution License, which permits unrestricted use, distribution, and reproduction in any medium, provided the original work is properly cited.

This paper proposes a model to find the optimal location of autonomous vehicle lanes in a transportation network consisting of both Autonomous Vehicles (AVs) and Human-Driven Vehicles (HDVs) while accounting for the roadway capacity variation. The main contribution of the model is considering a generalized definition of capacity as a function of AV proportion on a link and incorporating it into the network design problem. A bilevel optimization model is proposed with total travel time as the objective function to be minimized. At the upper-level problem, the optimal locations of AV lanes are determined, and at the lower level which is a multiclass equilibrium assignment, road users including both AVs and HDVs seek to minimize their individual travel times. It is shown that if capacity variation is ignored, the effect of AV lane deployment can be misleading. Since there will be a long transition period during which both AVs and HDVs will coexist in the network, this model can help the network managers to optimally reallocate the valuable road space and better understand the effects of AV lane deployment at the planning horizon as well as during the transition period. Employing this model as a planning tool presents how the proposed AV lane deployment plan could consider the AV market penetration growth during the transition period. Numerical analysis based on the Sioux Falls network is presented in two cases with and without variable capacity to illustrate the application of this model. At the $60 \%$ penetration rate of AVs, the improvement in total travel time was $3.85 \%$ with a fix capacity while this improvement was $9.88 \%$ with a variable capacity.

\section{Introduction}

In recent years, Autonomous Vehicles (AVs) have received considerable attention from different research groups all over the world. They are expected to have potential benefits on safety, mobility, traffic efficiency, driver productivity, road capacity, and environment [1-4].

Despite significant advances in autonomous vehicle technology, there are still many stages and many years left to make driverless cars ready for broad adoption [5]. Considering the patterns of previous vehicle technology deployments such as automatic transmissions, airbags, and hybrid vehicles, it takes decades for autonomous vehicle technology to reach market saturation [5]. Reviewing studies about AV adoption [5-8] leads to the conclusion that there will be a long transition period in which AVs and human-driven vehicles (HDVs) share the road. Therefore, policymakers and practitioners should prepare strategies for the time that AVs and HDVs coexist and share the same transportation network. These strategies need to recognize different sources of uncertainty in transportation planning for AVs such as technology, adoption rates, roadway capacity, land use changes, and travel demand [9].

Due to the high uncertainty in the penetration rate of AVs, flexible and low-cost strategies are required. Among available strategies, providing dedicated lanes to autonomous vehicles using existing infrastructure is a flexible and low-cost one. It is a tactical decision which can benefit the network through existing resources [10]. However, conversion of a general purpose lane to a dedicated lane may lead to public dissatisfaction. A 
dedicated AV lane is one of the managed lane strategies with a similar concept to High Occupancy Vehicle (HOV) lanes, High Occupancy Toll (HOT) lanes, and express lanes. Previous experiences with these strategies show if they are designed and operated properly, they can improve safety, efficiency, and the environment quality [11]. Despite the similarities between dedicated AV lanes and other managed lane strategies, there are yet differences such as interactions between AVs and HDVs, which make it necessary to specifically examine the adaptation of dedicated AV lanes.

Some studies suggest that many potential AV benefits require platooning, which can be achieved by dedicating lanes to AVs [5]. On the other hand, effective platooning and consequently successful implementation of dedicated AV lanes highly depends on their market penetration rate; for example, at low market penetration rates benefits may not be significant [12]. Several studies have used simulation models to investigate the effect of a dedicated AV lane on traffic flow. Microscopic simulation models are one of the tools to consider the interactions between AVs and HDVs. Talebpour et al. [12] developed a microscopic simulation model to explore how congestion and travel time reliability are affected by reserving a lane for AVs on a hypothetical twolane segment and a four-lane highway segment. Their results indicate that optional use of dedicated lanes by AVs can improve link performance both in terms of congestion and travel time reliability while limiting AVs to dedicated lanes decreases the overall performance. Vander Laan and Sadabadi [13] used macroscopic traffic simulation to address effects of a dedicated AV lane on the performance of a congested corridor. They applied it to a four-lane corridor with one AV lane. Their results showed that the best system performance is obtained at medium market penetration rates (i.e., 30\%, 40\%, and 50\%). Ye and Yamamoto [14] reached a similar conclusion. They proposed a microscopic simulation model to evaluate the performance of overall traffic throughput under different AV market penetration rates while allocating a lane to connected and autonomous vehicles (CAVs) in a heterogeneous traffic flow. They found that the positive effects of CAV dedicated lanes emerge at medium market penetration rates. Ghiasi et al. [15] formulated the capacity of a one-lane highway shared by CAVs and HDVs considering stochastic headways between different vehicle types. They also presented a lane management model that determines the optimal number of CAV dedicated lanes on a highway segment while maximizing throughput.

While most studies have explored the effect of a dedicated AV lane in a highway segment, there are only a limited number of studies investigating these effects at the network level. To understand the effects of exclusive AV lanes at the network level, Ivanchev et al. [16] designed an agent-based simulation for the city of Singapore. They converted one regular lane of each highway segment to an exclusive AV lane. The results indicate that the introduction of AV lanes to all highway segments worsens the entire network performance compared to the network without $\mathrm{AV}$ lanes for all AV penetration rates. The reason may be that introducing AV lanes to all highway segments limits the access of HDVs to a large part of the network. Therefore, the optimal location of AV lanes should be determined in order to obtain benefits at the network level. Wu et al. [17] examined network flow pattern under user equilibrium (UE) and system optimum (SO) in a linear traffic corridor with dedicated AV expressways and nonautonomous local streets. They also developed a problem of AV expressway exit as an optimization bilevel problem and explored the effect of exit locations on UE and SO patterns. Chen et al. [18] developed a mathematical framework which determined the optimal location, the time to deploy, and the number of AV lanes in a transportation network. They considered the capacity increase associated with AV shorter headways in an exclusive AV lane; however, capacity impacts of the presence of AVs in the traffic flow have been ignored. In fact they did not consider that the capacity of a roadway shared by AVs and HDVs is different from the capacity of the same roadway with homogeneous traffic flow of AVs or HDVs.

The approach used in previous studies [18] may lead to inaccurate conclusions as we show in this study. To avoid such inexact results, we have introduced the capacity function of mixed traffic to the lower level of the network design problem investigated in this paper.

To bridge this research gap, this study aims to develop a mathematical model that determines the optimal location of AV lanes in a network accounting for capacity changes of a roadway shared by AVs and HDVs. We considered capacity of each link as a function of link autonomy level which is the proportion of AVs on a link to total link flow. We call this approach variable capacity case, which can be compared against the fixed capacity case (i.e., the problem solved by [18]). This problem is designed as a network design problem in which at the upper level, the location of AV lanes are determined. The lower-level problem is a multiclass equilibrium assignment in which both AVs and HDV sollow the Wardrop equilibrium principle to minimize their individual travel costs. No study to date has formulated a network design problem for a network with mixed traffic of AVs and HDVs while examining the effects of capacity variations associated with presence of AVs.

The main advantage of the proposed model is considering a more realistic capacity function which is already developed in previous link level studies $[15,19]$ to the network level study by applying it in traffic assignment and the network design problem. The proposed change improves the estimated travel time accuracy as well as link flows in a network. To better explain the concept and contribution of this study, an analysis on a small network of Figure 1 is provided below.

Consider the network of Figure 1. Let $w$ be the set of origin-destination (OD) pairs. There are two OD pairs, $w=\{(1,3),(1,4)\}$. The demand of these OD pairs are $d_{13}=$ 3000 and $d_{14}=2000$ vehicles per hour. Assume all links have one lane and the AV penetration rate is $50 \%$ for both OD pairs. AVs and HDVs are allowed on all links. The BPR function of equation (1) is used to estimate the link travel time: 


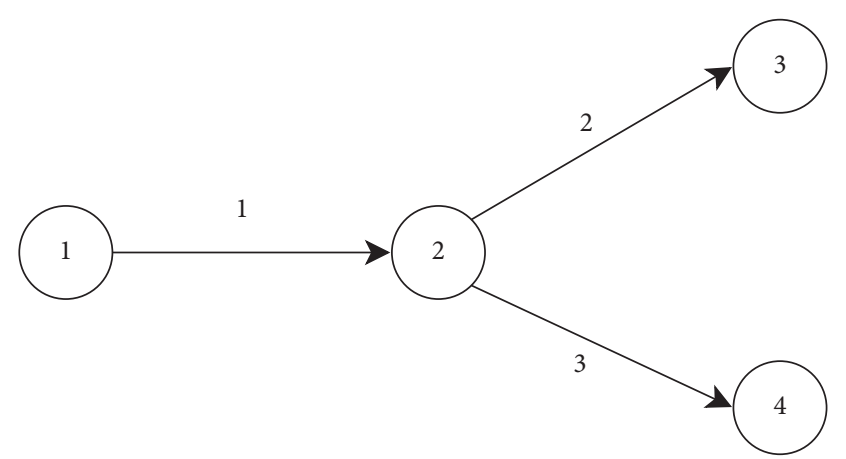

FIgURE 1: A simple network to illustrate the study contribution.

$$
t_{a}=t_{a}^{0}\left(1+0.15\left(\frac{V_{a}}{C_{a}}\right)^{4}\right)
$$

where $t_{a}$ is the link travel time, $t_{a}^{0}$ is the link free-flow travel time which is assumed to be 6 minutes for all links of this example, and $V_{a}$ and $C_{a}$ are link flow and capacity, respectively. We computed total travel time at equilibrium for two cases: the fixed capacity case and the variable capacity case (i.e., the approach of this study). Per-lane capacity for the fixed capacity case is assumed to be 2400 vehicles and for the variable capacity case is computed by equation (2) [16], where $h_{\mathrm{AV}}$ and $h_{\mathrm{HDV}}$ are headway of AVs and HDVs to a leading vehicle, respectively, and $p_{a}$ is proportion of AVs on a link:

$$
C_{a}=\frac{3600}{h_{\mathrm{AV}} p_{a}+h_{\mathrm{HDV}}\left(1-p_{a}\right)}
$$

Let $h_{\mathrm{AV}}=0.85$ and $h_{\mathrm{HDV}}=1.5$, the total travel time is equal to 15,231 vehicle minutes for the first case and 61,341 for the second case. The results indicate that integrating capacity variations into the link performance function improves the accuracy of travel time estimation. Since traffic assignment is the lower-level problem of a network design problem, the travel time estimation affects the optimal solution too.

The remainder of this paper is divided into five sections. Section 2 provides a brief review of studies related to our work. Section 3 proposes the mathematical framework, and Section 4 explains the solution method used to solve the problem. In Section 5, results of numerical analysis are presented. Section 6 concludes the paper.

\section{Literature Review}

In this section, studies related to this work is summarized in three main topics: (a) network design problems solved for AVs so far, (b) equilibrium assignment problem in transportation networks with AVs and HDVs, and (c) capacity impacts of AVs in a heterogeneous traffic flow (mix of AVs and HDVs). Little research has been conducted on the first and second topics. However, the last issue has drawn the main attention from researchers.
2.1. Network Design Problem (NDP) in the Context of Autonomous Vehicles. NDP is a well-known optimization problem in transportation planning literature, which aims to find the optimal value of specific objectives (e.g., total travel time) under limited resources. The ultimate goal of the NDP is to determine optimal projects among a set of alternatives while accounting for route choice behavior of road users. NDP can be considered as a two-stage leaderfollower Stackelberg game which is usually formulated as a bilevel problem [20]. At the upper level, the network manager (leader) aims to optimize network performance under resource constraints, and at the lower level, the network users (followers) choose a route with the minimum travel cost.

Research on a network design problem for different transportation modes with various objective functions, decision variables, and a wide range of solution methods has a long history in transportation [21, 22]. However, a few network design problems have considered the context of AVs.

Chen et al. [18] proposed an optimization model for $\mathrm{AV}$ lane deployment on a transportation network in a mixed AV and HDV environment. The upper-level problem determined the deployment plan of AV lanes to minimize the social cost while considering a diffusion model to forecast the AV adoption rate. The lower-level problem was a multiclass user equilibrium which followed Wardrop's first equilibrium principle [23]. The objective function was the total social cost, which included the value of travel time for both modes and the cost related to the unsafety factor for using HDVs in the planning horizon. They used the US Bureau of Public Roads (BPR) formula as a volume-delay function while assuming a fix per-lane capacity for regular links in the network. The per-lane capacity for AV links was assumed fixed and greater than the per-lane capacity of the adjacent regular link, given that AVs can achieve shorter headways compared to HDVs. Applying the proposed model to the South Florida network showed that there was no considerable gain from deploying AV lanes at low market penetrations (e.g., less than 20\%). Furthermore, the optimal deployment plan was at the 25 th year of a 40 year horizon where the social cost was reduced by $3.91 \%$.

Another related network design problem is the optimal design of AV zones in transportation networks [24]. The problem was expressed as a mixed integer bilevel model that determined the optimal AV zones in a general network while both AVs and HDVs followed the user equilibrium outside the AV zones. AVs assumed to follow the system optimum within the AV zones. In their study, the AV zone is defined as a zone with links dedicated to AVs. Similar to their previous study, it was assumed that the capacity of links within an AV zone was more than mixed AV and HDV links and the capacity of links with mixed AV and HDV and HDV only links were equal. Numerical analyses showed that designing AV zones in a transportation network reduced the total travel cost. 
2.2. User Equilibrium Assignment Problem in Mixed Traffic. Static equilibrium assignment is the most common problem used as the lower level of the NDP. Although many researchers have studied the problem properties, formulation, extensions, and solution methods since the introduction of Wardrop principles in 1952 [23], there is little literature on the static equilibrium assignment with mixed autonomy to the best of the authors' knowledge. Of course, the main reason is the emerging nature of the subject. It will be of particular importance to formulate the equilibrium assignment problem appropriately when solving an NDP where AVs and HDVs share same roads, and all network users have a selfish route behavior. The mixed autonomy traffic assignment is a multiclass equilibrium model with special properties. The multiclass user model, which was formulated by Dafermos [25] is more complex than a single user model. The single user model can be formulated as a convex optimization model with a unique solution under the assumption of separable and increasing link performance function [26]. However, there exists no convex formulation for a general network with multiple classes of vehicles. In addition, class-specific link flow solution is not unique for a general network. However, the total flow of each link is unique in certain cases. For instance, with a BPR volume-delay function, fixed capacity for all links, and same capacity for all vehicle classes, uniqueness of total link flows is guaranteed [27].

Levin and Boyles [28] is the first study that accounted for roadway capacity changes in the presence of AVs in a traffic assignment model. They developed a four-stage travel demand model to investigate the effects of AV ownership on travel behavior. They used a static assignment model to identify the route choice behavior of multiple classes of road users, which are divided according to their value of time. The travel time of their model was based on the BPR function with capacity specified as a linear function of the jam density as defined in Greenshield's model (equation (3). In this study, jam density is defined by equation (4) as a function of the proportion of AVs on the link:

$$
\begin{aligned}
C_{a}\left(x_{a}\right)= & \rho k_{a}\left(x_{a}\right), \\
k_{a}\left(x_{a}\right)= & \frac{5280}{l_{H}}\left(\frac{\sum_{y \epsilon Y}\left(x_{a}^{y}\left(1-\Gamma_{\mathrm{AV}}^{y}\right)\right)}{\sum_{y \epsilon}\left(x_{a}^{y}\right)}\right) \\
& +\frac{5280}{l_{A}}\left(\frac{\sum_{y \epsilon Y}\left(x_{a}^{y}\left(\Gamma_{\mathrm{AV}}^{y}\right)\right)}{\sum_{y \epsilon}\left(x_{a}^{y}\right)}\right),
\end{aligned}
$$

where $C_{a}\left(x_{a}\right)$ is the capacity, $k_{a}\left(x_{a}\right)$ is the jam density of link $a$ in units of vehicles per mile, $\rho$ is the calibration constant, and $l_{H}$ and $l_{A}$ are the maintained vehicle spacing for HDVs and AVs, respectively. $x_{a}^{y}$ is the flow of class $y$ and $\Gamma_{\mathrm{AV}}^{y}$ is a Boolean variable indicating if class $y$ uses AVs entirely or not. Under the assumption $2 l_{A}>l_{H}$, the resulting volume-delay function satisfies the monotonicity condition with respect to each single class which is the necessary condition (but not sufficient) for the convexity of a multiclass problem. The Frank Wolfe algorithm was used to solve the equilibrium problem applied to Austin, Texas, and downtown network. Although the results of the numerical analysis showed the convergence of the algorithm, Levin and Boyles mentioned that the algorithm may not converge, and multiple equilibria are certainly possible.

A recent study of Mehr and Horowitz [27] on the equilibrium state of traffic network in a mixed AV and HDV environment showed that introducing capacity impacts of autonomous vehicles in the mixed flow makes the multiclass equilibrium problem more complex. They found that the equilibrium problem does not have a unique solution in a general network with both AVs and HDVs. In this problem, neither class-specific link flows nor total link flows are unique. The results are based on BPR volume-delay function with the following form:

$$
t_{a}\left(f_{a}\right)=t_{a}^{0}\left(1+\gamma_{a}\left(\frac{f_{a}}{C_{a}\left(\alpha_{a}\right)}\right)^{\beta_{a}}\right)
$$

where $f_{a}$ is the total link flow of link $a, t_{a}^{0}$ is the free flow travel time, $\gamma_{a}$ and $\beta_{a}$ are nonnegative parameters, and $C_{a}\left(\alpha_{a}\right)$ is the capacity of link $a$ which is a function of the proportion of AVs on link $a$. Despite other available capacity functions for estimating link capacity in a mixed condition, which will be discussed later in Section 2.3, capacity was defined as follows:

$$
C_{a}\left(\alpha_{a}\right)=\frac{m_{a} M_{a}}{\alpha_{a} m_{a}+\left(1-\alpha_{a}\right) M_{a}}
$$

where $m_{a}$ and $M_{a}$ are the capacity of link $a$ when all vehicles on the link are HDVs and AVs, respectively $\left(m_{a}<M_{a}\right) . \mu_{a}$ is defined as the ratio of $m_{a}$ to $M_{a}$ and is conceptually the degree of capacity asymmetry of link $a$.

Mehr and Horowitz proved that using the volume-delay function of form (5) and the capacity function of form (6), as well as assuming a homogeneous degree of capacity asymmetry (i.e., same $\mu_{a}$ for all links) in a general network with a fixed AV market penetration, the uniqueness of total travel time is guaranteed for all Wardrop equilibrium flow vectors.

Wang et al. [29] developed a static multiclass traffic assignment model for a mixed AV and HDV environment. Their proposed model offers a different type of route choice behavior for each group of road users. AV users are assumed to follow the user equilibrium principle while HDV users are assumed to choose their route according to a crossnested logit model. They considered the fact that AVs could have lower reaction time than HDVs which can lead to an increased link capacity. To estimate link travel time, they used the BPR function with the link capacity formulated as follows:

$$
C_{a}=\frac{1}{\left(v_{a, H} / v_{a, H}+v_{a, A}\right)\left(1 / C_{a, H}\right)+\left(v_{a, A} / v_{a, H}+v_{a, A}\right)\left(1 / C_{a, A}\right)},
$$

where $C_{a}$ is the capacity of $\operatorname{link} a, C_{a, A}$ and $C_{a, H}$ are the capacity of link $a$ when all vehicles on the link are AVs and HDVs, respectively, and variables $v_{a, A}$ and $v_{a, H}$ are the AV and HDV flows on link $a$. They have applied a new routeswapping-based solution algorithm to solve the problem. The suggested algorithm is path-based and can only find one local optimal depending on the initial path flow pattern. 
2.3. Capacity Impacts of Autonomous Vehicles in the Heterogeneous Traffic Flow. Several studies suggest that AVs can improve roadway capacity through the possibility of platooning and shorter safe-intervehicle distance compared to HDVs. Tientrakool et al. [30] compared the highway capacity of three vehicle technologies: manual vehicles, vehicles with sensors, and communicating vehicles. The results indicated that when all vehicles were equipped with sensors, the capacity was about 1.5 times of when all vehicles were manual. Furthermore, communicating vehicles could lead to a $237 \%$ increase in the highway capacity.

Chen et al. [31] proposed an analytical formulation for capacity of a single-lane and a two-lane highway with heterogeneous traffic of both AVs and HDVs. They extend their formulation to a general case of a multilane highway. According to their study, the AV penetration rate, platoon size, and spacing characteristics were the parameters that affect capacity. They also proposed capacity functions under different lane policies including exclusive AV or HDV lanes and mixed-used lanes. They found that policies that segregate AVs and HDVs (i.e., have exclusive $\mathrm{HDV}$ and $\mathrm{AV}$ lanes), led to a lower capacity than policies including mixedused lanes.

Ghiasi et al. [15] formulated a mixed traffic capacity as a function of the AV market penetration, platooning intensity, and mixed traffic headway settings based on a Markov chain model. They showed that unlike the generally accepted assumption, higher market penetration and platooning intensity did not necessarily lead to higher capacity, since headway setting is dependent on the AV technology and plays an important role in determining the capacity.

Lazar et al. [19] proposed two capacity models under two different scenarios. The main assumption in the first scenario was that AVs are capable of maintaining reduced headways regardless of the preceding vehicle. The second scenario assumed that the headway depends on the preceding vehicle, and a short headway is possible when an $\mathrm{AV}$ follows another AV. In this case, the distribution of vehicles on the road is important. This study presented a capacity formulation assuming that vehicle types are determined as a result of the Bernoulli process. In both scenarios, capacity was the function of proportion of AVs on the road. The capacity calculated in the first scenario served as an upper bound for the capacity in the second scenario [32].

\section{Problem Statement and Formulation}

In this study, we consider a network with two types of lanes: regular lanes and AV lanes. Two modes use the transportation network: AVs and HDVs. AVs can use all lanes in the network and HDVs can only use regular lanes. The demand for each origin-destination (O-D) pair is assumed fixed for each travel mode. The route choice behavior of both modes is based on Wardrop's first principle.

We formulated AV lane location problem as an integer bilevel programming (IBP). The IBP consists of optimally selected lanes from the existing network to be dedicated to AVs. Below we summarize the notations used in the model.
Sets:

$N$ : set of nodes

$A$ : set of links

$\widetilde{A}$ : set of candidate AV links

$K$ : set of paired links

$M$ : set of travel modes: mode 1 denotes HDVs and mode 2 denotes AVs

$W$ : set of origin-destination (OD) pairs

\section{Parameters:}

$m$ : index of travel mode, $m \in M$

$w$ : index of OD pair, $w \in W$

$d^{w, m}$ : demand of travel mode $m \in M$ between OD pair $w \in W$

$\bar{A}$ : the node-link incidence matrix with elements $\alpha_{n a}$, $n \in N, a \in A . \bar{A}=\left\{\alpha_{n a}\right\}$, if link $a$ starts in node $n$, $\alpha_{n a}=1$; if link $a$ ends in node $n, \alpha_{n a}=-1$; otherwise $\alpha_{n a}=0$

$D^{w, m}:$ the demand vector with $d_{n}^{w, m}$, $n \in N, w \in W, m \in M . D^{w, m}=\left\{d_{n}^{w, m}\right\}$, if node $n$ is the origin of $w, d_{n}^{w, m}=d^{w, m}$; if node $n$ is the destination of $w, d_{n}^{w, m}=-d^{w, m} ;$ otherwise $d_{n}^{w, m}=0$

$L_{a}$ : number of lanes on link $a \in A$

$\xi$ : capacity reduction factor in mixed traffic

$h_{\mathrm{AV}}$ : headway of AVs to a leading vehicle

$h_{\mathrm{HDV}}$ : headway of HDVs to a leading vehicle

$d_{a}^{k}$ : if link $a$ belongs to the $k$ th link pair, and it is an AV link, $d_{a}^{k}=1$; if it is a regular link, $d_{a}^{k}=-1$; otherwise $d_{a}^{k}=0$

Variables:

$x_{a}^{w, m}$ : flow of travel mode $m \in M$ on link $a \in A$ between OD pair $w \in W$

$x_{a}$ : aggregate flow on link $a \in A$

$y^{k}$ : if one lane of the $k$ th link pair is converted to an

AV lane, $y^{k}=1$; otherwise $y^{k}=0$

$C_{a}$ : total capacity of link $a \in A$

$\bar{c}_{a}$ : per-lane capacity of link $a \in A$

$p_{a}$ : proportion of AVs on link $a \in A$

3.1. Network Representation. Let $G(N, A)$ represent a general transportation network with node set $N$ and link set $A$. Define $\widetilde{A}$ as a set of candidate links to introduce an AV lane $(\widetilde{A} \subset A)$. Each candidate AV link is paired with a regular link. Candidate links are links with more than two lanes because there should be at least one regular lane between each OD pair to maintain network connectivity. Figure 2 illustrates a sample network topology. In Figure 2, the solid line represents regular links, and the dotted line denotes the candidate AV links. The set of paired links is defined as $K$. Specifically, in Figure 2, $A=\{1,2,3,4,5\}, \widetilde{A}=\{4,5\}$, and $K=\{(1,4),(2,5)\}$. In this example, links 1 and 2 have two lanes and link 3 has just one lane, so no paired AV link is defined for link 3.

3.2. Multiclass User Equilibrium Problem. An equilibrium model for a general multiclass transportation network can be 


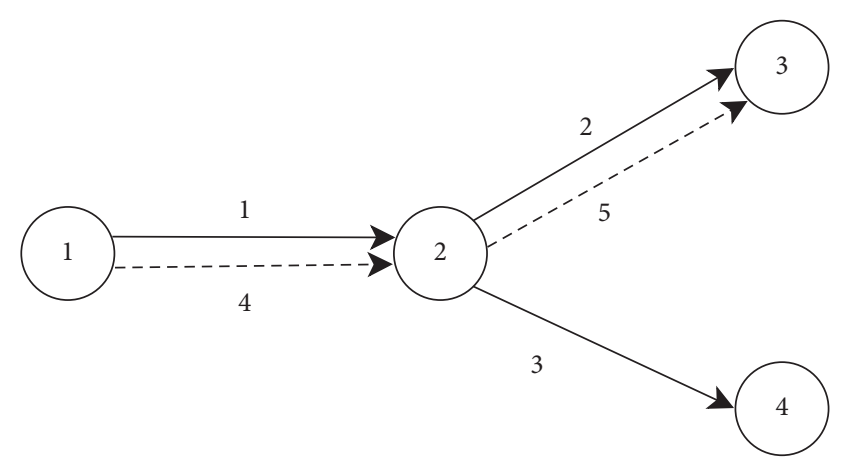

Figure 2: An example of network topology.

written as a Variational Inequality (VI) problem of equation (8) $[33,34]$. The solution of the following VI formulation with the feasible region in $\Omega$ is $\mathbf{x}^{*}$. It can be shown that $\mathbf{x}^{*}$ satisfies the multiclass user equilibrium condition as defined by Dafermos [25]:

$$
\sum_{w} \sum_{m} \sum_{a} t_{a}\left(x^{*}\right)\left(x_{a}^{w, m}-x_{a}^{w, m *}\right) \geq 0, \quad \forall x \in \Omega .
$$

The feasible region $\Omega$ in a multiclass transportation network with exclusive AV lanes is mathematically described by equations (9)-(13). Constraint (9) simply ensures the conservation of flow in the network. Constraint (10) is defined on sets of regular links (i.e., $A \backslash \widetilde{A}$ ). Nonnegativity of flows is expressed by (10) and (12). By (10) and (11), we allow HDVs on regular links, and by (12) we allow AVs on both lane types. Constraint (13) defines aggregate link flow as the sum of link flows across all modes and O-D pairs:

$$
\begin{gathered}
\bar{A} \mathbf{x}^{w, m}=D^{w, m}, \quad \forall w \in W, \forall m \in M, \\
x_{a}^{w, 1} \geq 0, \quad \forall a \in A \backslash \widetilde{A}, \forall w \in W, \\
x_{a}^{w, 1}=0, \quad \forall a \in \widetilde{A}, \forall w \in W, \\
x_{a}^{w, 2} \geq 0, \quad \forall a \in A, \forall w \in W, \\
x_{a}=\sum_{m \in M} \sum_{w \in W} x_{a}^{w, m}, \quad \forall a \in A .
\end{gathered}
$$

The link performance function, which calculates travel time, is the BPR function in the form of equation (14), where $t_{a}^{0}$ is the free flow travel time of link $a$ and $C_{a}$ is the capacity of link $a$. In this study, we assume that the capacity of each link is the per-lane capacity times the number of lanes (equation (15). The per-lane capacity is considered similar to that of Mehr and Horowitz [27] (equation (16) with homogeneous degree of capacity asymmetry (i.e., for all links $\left(h_{\mathrm{AV}} / h_{\mathrm{HDV}}\right)$ is constant).
This function gives the lane capacity based on the share of AVs in the flow and also ensures that a unique total travel time is resulted despite the fact that multiple Wardrop equilibria might exist. According to Lazar et al. [19], the proposed capacity function gives an upper bound for capacity; thus, we consider $\xi$ as a capacity reduction factor in mixed traffic. It is a realistic assumption since it is unlikely that shared use lanes reach their maximum capacity due to complex interactions between AVs and HDVs. $\xi$ is a positive coefficient less than 1.0 for shared use lanes and is equal to 1.0 for exclusive AV lanes. Link autonomy level which is defined as the share of AVs on a link is calculated by equation (17):

$$
\begin{aligned}
t_{a}\left(x_{a}, C_{a}\right) & =t_{a}^{0}\left(1+0.15\left(\frac{x_{a}}{C_{a}\left(p_{a}\right)}\right)^{4}\right), \\
C_{a}\left(p_{a}\right) & =\bar{c}_{a}\left(p_{a}\right) L_{a}, \\
\bar{c}_{a}\left(p_{a}\right) & =\frac{3600 \xi}{h_{\mathrm{AV}} p_{a}+h_{\mathrm{HDV}}\left(1-p_{a}\right)}, \\
p_{a} & =\frac{\sum_{w \in W} x_{a}^{w, 2}}{\sum_{w \in W} x_{a}^{w, 1}+\sum_{w \in W} x_{a}^{w, 2}} .
\end{aligned}
$$

3.3. A V Lane Location Problem. The problem of determining the optimal location of AV lanes in the network is a bilevel program in which the lower level is a multiclass user equilibrium expressed by equations (8)-(13), and the upperlevel problem finds the AV lane location in the network to minimize the total travel cost. The upper-level problem can be formulated as follows.

Integer bilevel program:

$$
\min _{x, y} \sum_{a \in A} t_{a}\left(x_{a}\right) x_{a}
$$

s.t.

$$
\begin{gathered}
C_{a}=\bar{c}_{a}\left(L_{a}+\sum_{k \in K} d_{a}^{k} y^{k}\right), \quad \forall a \in A, \\
y^{k} \in\{0,1\}, \quad \forall k \in K .
\end{gathered}
$$

Objective (18) is to minimize the total travel time of the transportation network where $x_{a}$ is determined by the lower level user equilibrium model. A binary variable $y^{k}$ is introduced to indicate whether an AV candidate link is converted to AV link or not. $d_{a}^{k}$ is defined as a parameter which can take three values as below:

$$
d_{a}^{k}=\left\{\begin{array}{cl}
1, & \text { if link a belongs to the } k \text { th link pair and it is an AV link } \\
-1, & \text { if link a belongs to } k \text { th link pair and it is a regular link } \\
0, & \text { else. }
\end{array}\right.
$$


The capacity of link $a$ is calculated by equation (19).

In brief, the problem is to find the optimal location of $\mathrm{AV}$ lanes in a network with mixed traffic flow of AVs and HDVs while minimizing the total travel time as the objective function and accounting for capacity variation. Thus, given the transportation network with a specified demand matrix, the output of the model is the optimal location of AV lanes. Below, we summarize basic assumptions of our model:

(i) A link with two lanes or more can be a candidate of an AV lane

(ii) Both road users choose their routes based on the user equilibrium principle

(iii) The link travel time is estimated by the BPR function with the variable capacity formulated by equation (16)

(iv) The ratio of $h_{\mathrm{AV}}$ (i.e., headway of $\mathrm{AVs}$ to a leading vehicle) to $h_{\mathrm{HDV}}$ (i.e., headway of HDV s to a leading vehicle) is the same for all network links

\section{Solution Method}

The transportation network design problem (TNDP) is NPhard even in the simplest form [35]. Several solution methods, such as exact methods, heuristics, and metaheuristics have been developed to solve the TNDP $[22,36,37]$. Hybrid heuristics and metaheuristics approaches are other methods that can be used to solve network design problems. The exact methods such as the branch and bound algorithm proposed by Leblanc [38] will become computationally inefficient in case of large-size networks due to its high accuracy. On the contrary, heuristics and metaheuristics are more efficient but less accurate methods. Hybrid methods which combine the two methods are more efficient than one heuristic or metaheuristic algorithm, as shown by some studies [39-41]

To solve the bilevel problem proposed in this study, we applied a heuristic method proposed by Bagloee et al. [42] which is a hybrid machine learning and optimization. The method employs a multivariate linear regression and an integer linear program to solve the bilevel problem iteratively. The algorithm starts with a feasible solution. Then, the traffic assignment problem (i.e., the lower-level problem) is solved based on this solution, and the objective function of the upper-level problem is computed. The objective function is regressed against the decision variables, and the coefficients of decision variables are calibrated. The next step is to solve an integer linear program (ILP), which finds a new feasible decision variable for the next iteration. The lowerlevel problem is solved based on the new solution, and a new regression is applied to the updated data. The termination criterion is the number of iterations, which is a user-specified parameter. The algorithm is applicable to large scale networks and can quickly converge to a local optimum [42].

Let

$$
\begin{aligned}
& i \text { : iteration counter } \\
& i_{\max }: \text { maximum number of iterations }
\end{aligned}
$$

$y_{i}^{k}$ : the feasible solution at iteration $i$

$Z_{i}$ : the objective function value at iteration $i$

The proposed algorithm is as follows [42]:

Step 0 : specify $i_{\max }$; set $i=0$; set $y_{0}^{k}=0$ as the initial feasible solution.

Step 1: solve the traffic assignment problem for the given $y_{i}^{k}$ and compute the objective function $Z_{i}$.

Step 2: estimate new regression coefficients of equation (18) using $y_{i}^{k}$ and the corresponding $Z_{i}$ :

$$
\bar{Z}_{i}=\sum_{k \in K} b^{k} y^{k}+b^{0}
$$

Step 3: update the ILP of equations (19)-(25) according to the current binary solution $y_{i}^{k}$ and estimated coefficients:

$$
\min _{y^{k}} \sum_{k} b^{k} y^{k}
$$

$$
\text { s.t. } y^{k} \in\{0,1\},
$$

$$
\begin{gathered}
\sum_{k \in Y_{1}^{j}} y^{k}-\sum_{k \in Y_{0}^{j}} y^{k} \leq\left|Y_{1}^{j}\right|-1, \quad \text { where } Y_{1}^{j}=\left\{k \mid y_{j}^{k}=1\right\} ; \\
Y_{0}^{j}=\left\{k \mid y_{j}^{k}=0\right\}, \quad j=1, \ldots, i .
\end{gathered}
$$

Step 4: if the termination criterion is met (i.e., $i=i_{\max }$ ), stop and report the best objective function $\left(Z_{i}\right)$ and corresponding $\left(y_{i}^{k}\right)$ as the final solution, else, solve the updated ILP in step 3, introduce $y_{i+1}^{k}$ as a new feasible solution, set $i=i+1$, and go to Step 1 .

\section{Numerical Analysis}

5.1. Basic Settings. The performance of the proposed model on the Sioux Falls network is presented in this section. The network has 76 links and 24 nodes and its data is available at a repository maintained by Bar-Gera [43] including node coordinates, start node, and end node of each link, capacity, length, and free flow travel time of each link. The given OD flows are 0.1 of the original daily flows in LeBlanc et al. [43]. There is no explicit data on the number of lanes of each link. Therefore, we have converted the given capacity to the number of lanes assuming that capacity is defined for the same period as OD flows and per-lane capacity of each link is 2400 vehicles per hour. Table 1 shows the results of the calculation. Note that the values calculated for number of lanes are rounded up to the nearest integer. Since the only constraint for a link to be a candidate of introducing an AV lane is to have more than one lane, there are 16 two way links that make our alternative set. Dotted links in Figure 3 are the candidates to get an AV lane. In Figure 3, both directions of a link are considered as one alternative (e.g., $(1,2)$ and $(2,1)$ is considered as one alternative to get an exclusive AV lane or not). Note that each candidate for an AV lane is paired with a 
TABLE 1: Number of lanes on each link.

\begin{tabular}{|c|c|c|c|}
\hline $\begin{array}{l}\text { Start } \\
\text { node }\end{array}$ & $\begin{array}{l}\text { End } \\
\text { node }\end{array}$ & $\begin{array}{c}\text { Capacity (vehicle per } \\
\text { hour) }\end{array}$ & $\begin{array}{c}\text { Number of } \\
\text { lanes }\end{array}$ \\
\hline 1 & 2 & 10791.75 & 5 \\
\hline 1 & 3 & 9751.45 & 5 \\
\hline 2 & 1 & 10791.75 & 5 \\
\hline 2 & 6 & 2065.91 & 1 \\
\hline 3 & 1 & 9751.45 & 5 \\
\hline 3 & 4 & 7129.38 & 3 \\
\hline 3 & 12 & 9751.45 & 5 \\
\hline 4 & 3 & 7129.38 & 3 \\
\hline 4 & 5 & 7409.50 & 4 \\
\hline 4 & 11 & 2045.34 & 1 \\
\hline 5 & 4 & 7409.50 & 4 \\
\hline 5 & 6 & 2061.66 & 1 \\
\hline 5 & 9 & 4166.67 & 2 \\
\hline 6 & 2 & 2065.91 & 1 \\
\hline 6 & 5 & 2061.66 & 1 \\
\hline 6 & 8 & 2041.08 & 1 \\
\hline 7 & 8 & 3267.42 & 2 \\
\hline 7 & 18 & 9751.45 & 5 \\
\hline 8 & 6 & 2041.08 & 1 \\
\hline 8 & 7 & 3267.42 & 2 \\
\hline 8 & 9 & 2104.25 & 1 \\
\hline 8 & 16 & 2102.43 & 1 \\
\hline 9 & 5 & 4166.67 & 2 \\
\hline 9 & 8 & 2104.25 & 1 \\
\hline 9 & 1 & 5798.25 & 3 \\
\hline 10 & 9 & 5798.25 & 3 \\
\hline 10 & 11 & 4166.67 & 2 \\
\hline 10 & 15 & 5630.00 & 3 \\
\hline 10 & 16 & 2022.88 & 1 \\
\hline 10 & 17 & 2080.63 & 1 \\
\hline 11 & 4 & 2045.34 & 1 \\
\hline 11 & 1 & 4166.67 & 2 \\
\hline 11 & 12 & 2045.34 & 1 \\
\hline 11 & 14 & 2031.88 & 1 \\
\hline 12 & 3 & 9751.45 & 5 \\
\hline 12 & 11 & 2045.34 & 1 \\
\hline 12 & 13 & 10791.75 & 5 \\
\hline 13 & 12 & 10791.75 & 5 \\
\hline 13 & 24 & 2121.36 & 1 \\
\hline 14 & 11 & 2031.88 & 1 \\
\hline 14 & 15 & 2136.47 & 1 \\
\hline 14 & 23 & 2052.00 & 1 \\
\hline 15 & 1 & 5630.00 & 3 \\
\hline 15 & 14 & 2136.47 & 1 \\
\hline 15 & 19 & 6068.65 & 3 \\
\hline 15 & 22 & 3999.66 & 2 \\
\hline 16 & 8 & 2102.43 & 1 \\
\hline 16 & 1 & 2022.88 & 1 \\
\hline 16 & 17 & 2179.13 & 1 \\
\hline 16 & 18 & 8199.96 & 4 \\
\hline 17 & 1 & 2080.63 & 1 \\
\hline 17 & 16 & 2179.13 & 1 \\
\hline 17 & 19 & 2009.98 & 1 \\
\hline 18 & 7 & 9751.45 & 5 \\
\hline 18 & 16 & 8199.96 & 4 \\
\hline 18 & 2 & 9751.45 & 5 \\
\hline 19 & 15 & 6068.65 & 3 \\
\hline 19 & 17 & 2009.98 & 1 \\
\hline 19 & 2 & 2084.42 & 1 \\
\hline
\end{tabular}

TABle 1: Continued.

\begin{tabular}{lccc}
\hline $\begin{array}{l}\text { Start } \\
\text { node }\end{array}$ & $\begin{array}{c}\text { End } \\
\text { node }\end{array}$ & $\begin{array}{c}\text { Capacity (vehicle per } \\
\text { hour) }\end{array}$ & $\begin{array}{c}\text { Number of } \\
\text { lanes }\end{array}$ \\
\hline 20 & 18 & 9751.45 & 5 \\
20 & 19 & 2084.42 & 1 \\
20 & 21 & 2108.30 & 1 \\
20 & 22 & 2114.87 & 1 \\
21 & 2 & 2108.30 & 1 \\
21 & 22 & 2179.13 & 1 \\
21 & 24 & 2035.57 & 1 \\
22 & 15 & 3999.66 & 2 \\
22 & 2 & 2114.87 & 1 \\
22 & 21 & 2179.13 & 1 \\
22 & 23 & 2083.33 & 1 \\
23 & 14 & 2052.00 & 1 \\
23 & 22 & 2083.33 & 1 \\
23 & 24 & 2116.05 & 1 \\
24 & 13 & 2121.36 & 1 \\
24 & 21 & 2035.57 & 1 \\
24 & 23 & 2116.05 & 1 \\
\hline
\end{tabular}

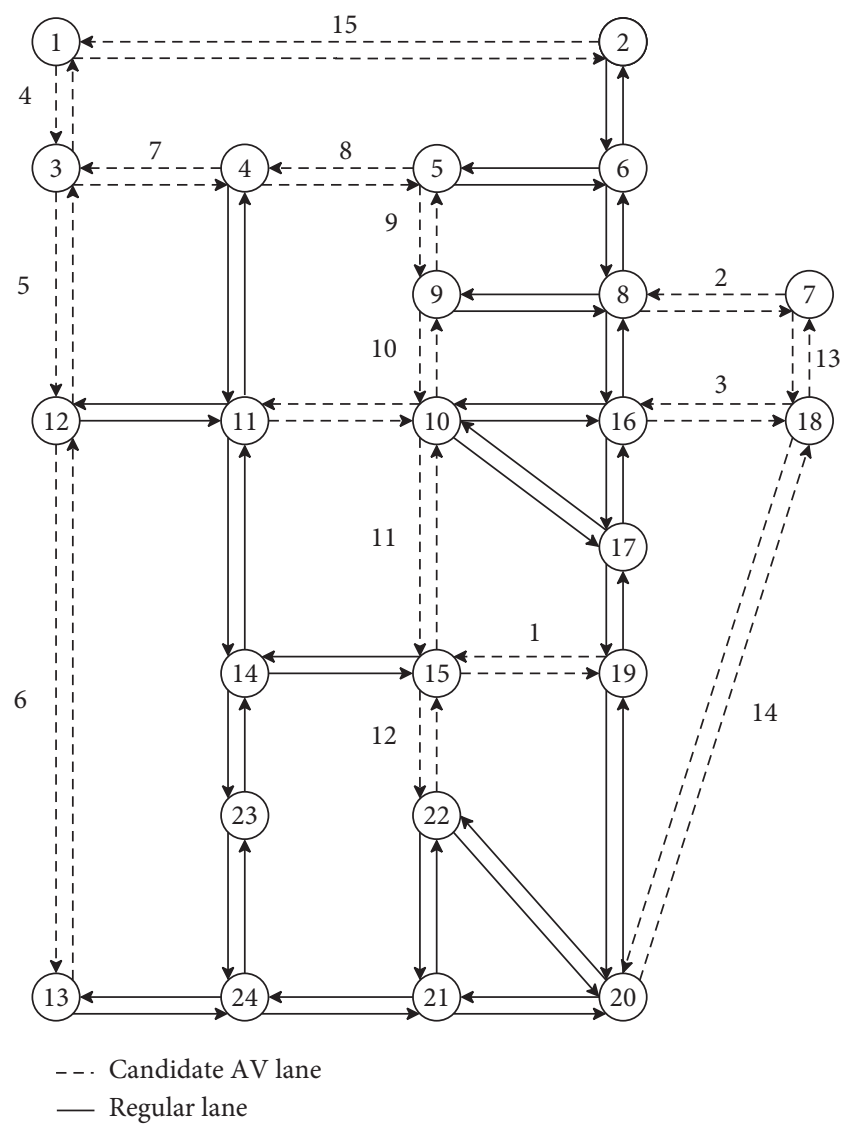

FIgURE 3: The Sioux Falls network, location of AV lane candidates.

regular link, as shown in Figure 2. The capacity reduction factor $(\xi)$ in mixed traffic is set to 0.8 for a mixed-used lane and 1 for an exclusive AV lane. The headway of AVs and HDVs from the preceding vehicle is 0.85 and 1.5 seconds, respectively. 
To solve the proposed bilevel problem, a code in python is developed which is synchronized to GAMS (the General Algebraic Modeling System), which is used to formulate the ILP problem as one part of the heuristic algorithm explained in Section 4. The rest part of the algorithm is coded in python.

5.2. Optimal Solution. During the transition period, market penetration rate grows from very low values to relatively high values until it reaches market saturation. Within the first stages of AV implementation, not much benefit would be obtained from the introduction of $\mathrm{AV}$ lanes to the network; however, more benefits are expected at higher market penetration rates. To approve this hypothesis we have conducted a sensitivity analysis on market penetration rates, the results of which are shown in Table 2. The benefits began to be significant at the $40 \%$ penetration rate, while improvements at lower rates (i.e., $10 \%, 20 \%$, and $30 \%$ ) are so marginal that a network manager may prefer not to implement any AV lanes. According to the analysis, an improvement in the objective function value was maximized at the market penetration rate of $60 \%$. The benefits of deploying AV lanes were reduced at both lower and higher rates. The optimal location plan at a market penetration rate of $50 \%$ is shown in Figure 4 as an example. This solution contains a selection of 12 alternatives out of 16 possible alternatives. The total travel cost at the optimal scenario was 89371 vehicle hours, which was 3.26 percent better than the base scenario (i.e., do nothing).

In addition to the optimal plan, a practical plan is also proposed. If we want to present a practical AV lane deployment plan to the network manager while accounting for growth in the AV market penetration, we should consider practical hassle of adding or removing an AV lane every year during the transition period. Investigating the results shows that there are common alternatives in the optimum plan of most market penetration rates. For instance, alternatives 1 and 10 are selected in all penetration rates above $20 \%$. Using this observation and taking into account the fact that little benefit is gained when the market penetration rate was below $40 \%$, it can be recommended to start the implementation of $\mathrm{AV}$ lanes when $40 \%$ of all vehicles on the network are autonomous. The optimal solution at $40 \%$ includes alternatives $1,7,8,10,11,12$, and 14 . We add alternatives $3,9,14$, and 16 at $50 \%$, and for $60 \%, 70 \%$, and $80 \%$ alternative 2 is added to the previous set of alternatives. When the AV market size grows to $90 \%$, fewer lanes need to be converted to exclusive AV lanes; therefore, alternatives 3,7 , and 8 were no longer selected to have an AV lane. Some alternatives that were present in the optimal plan are not considered in the practical plan to decrease the practical hassle of adding and removing AV lanes every year during the transition period. Running the traffic assignment and calculating the objective function for this deployment plan (see Table 3 ) indicates that there is a little difference between this plan and the optimal plan of Table 2; therefore, the network benefits from implementation of AV lanes are maximized with minimum disturbances to the network structure.
TABLE 2: The optimal plan for different market penetration rates (variable capacity).

\begin{tabular}{|c|c|c|}
\hline $\begin{array}{l}\text { Market } \\
\text { penetration rate }\end{array}$ & Optimal plan & $\begin{array}{l}\text { Improvement in the } \\
\text { objective function } \\
\text { (percent) }\end{array}$ \\
\hline 10 & 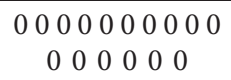 & 0.00 \\
\hline 20 & $\begin{array}{c}001001010000 \\
0011110\end{array}$ & 0.11 \\
\hline 30 & 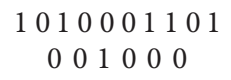 & 0.67 \\
\hline 40 & 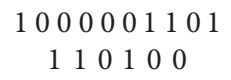 & 1.87 \\
\hline 50 & 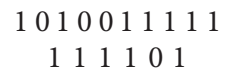 & 3.26 \\
\hline 60 & 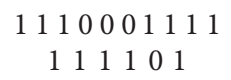 & 3.85 \\
\hline 70 & 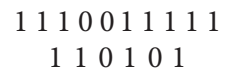 & 2.83 \\
\hline 80 & 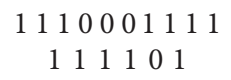 & 2.19 \\
\hline 90 & 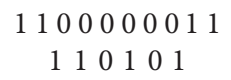 & 1.57 \\
\hline
\end{tabular}

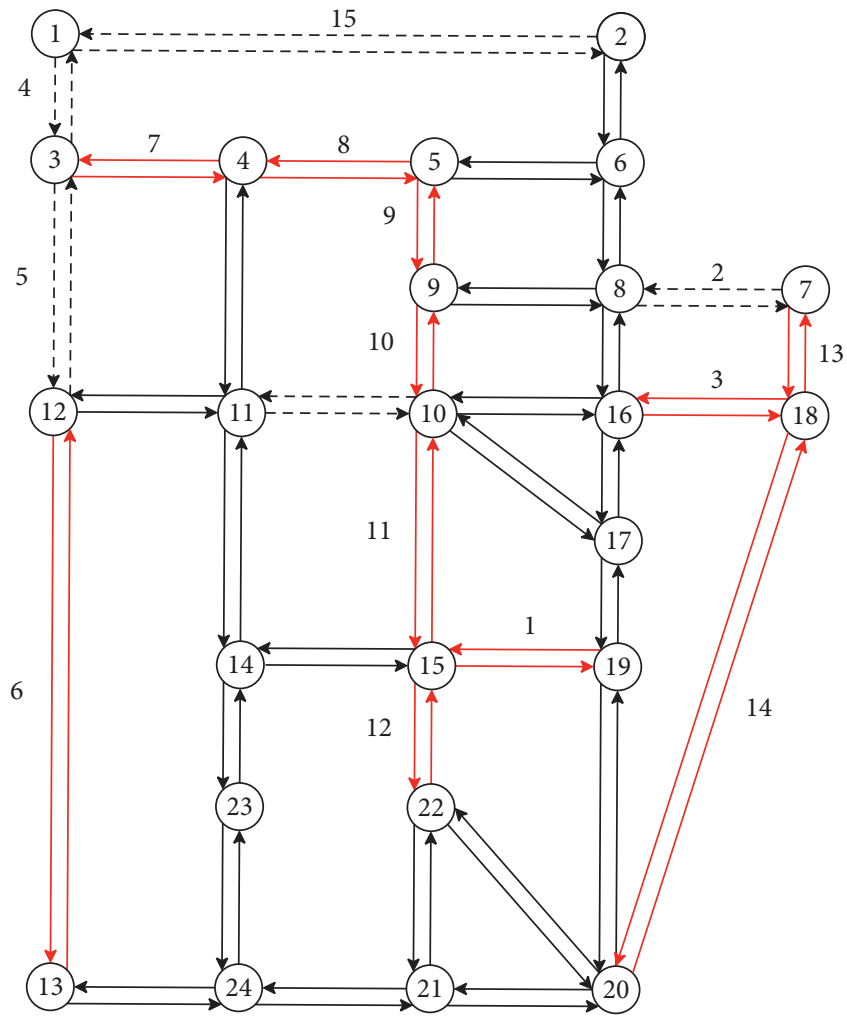

- - - Links that did not get an AV lane

- Links that were not candidates for an AV lane

_ Links that were introduced with an AV lane

FIgURE 4: Optimal plan at market penetration of $50 \%$.

In order to show the computational efficacy of the applied algorithm, the number of iterations and the CPU time to reach the optimal solution is presented in Table 4 . 
TABLE 3: The proposed practical plan.

\begin{tabular}{llllllllllllllllll}
\hline Market penetration rate & Proposed plan & Improvement difference with the optimal plan (percent) \\
\hline 10 & 0 & 0 & 0 & 0 & 0 & 0 & 0 & 0 & 0 & 0 & 0 & 0 & 0 & 0 & 0 & 0 & 0.000 \\
20 & 0 & 0 & 0 & 0 & 0 & 0 & 0 & 0 & 0 & 0 & 0 & 0 & 0 & 0 & 0 & 0 & -0.110 \\
30 & 0 & 0 & 0 & 0 & 0 & 0 & 0 & 0 & 0 & 0 & 0 & 0 & 0 & 0 & 0 & 0 & -0.670 \\
40 & 1 & 0 & 0 & 0 & 0 & 0 & 1 & 1 & 0 & 1 & 1 & 1 & 0 & 1 & 0 & 0 & 0.000 \\
50 & 1 & 0 & 1 & 0 & 0 & 0 & 1 & 1 & 1 & 1 & 1 & 1 & 1 & 1 & 0 & 1 & -0.003 \\
60 & 1 & 0 & 1 & 0 & 0 & 0 & 1 & 1 & 1 & 1 & 1 & 1 & 1 & 1 & 0 & 1 \\
70 & 1 & 0 & 1 & 0 & 0 & 0 & 1 & 1 & 1 & 1 & 1 & 1 & 1 & 1 & 0 & 1 \\
80 & 1 & 1 & 1 & 0 & 0 & 0 & 1 & 1 & 1 & 1 & 1 & 1 & 1 & 1 & 0 & 1 & -0.027 \\
90 & 1 & 1 & 0 & 0 & 0 & 0 & 0 & 0 & 1 & 1 & 1 & 1 & 1 & 1 & 0 & 1 & -0.003 \\
\hline
\end{tabular}

TABle 4: Number of iterations and CPU time to reach the optimal solution.

\begin{tabular}{lcc}
\hline Market penetration rate & Number of iterations to reach the optimal solution & CPU time (min) to reach the optimal solution \\
\hline 10 & 19 & 2.96 \\
20 & 35 & 6.76 \\
30 & 54 & 12.02 \\
40 & 46 & 11.52 \\
50 & 50 & 11.50 \\
60 & 65 & 15.10 \\
70 & 59 & 13.02 \\
80 & 48 & 11.46 \\
90 & 23 & 3.94
\end{tabular}

We have used a laptop computer with Intel Corei7 of $2.70 \mathrm{GHz}$ and $12 \mathrm{~GB}$ of RAM. It is worth noting that the optimal solution in all cases is the global optimal solution which is already identified by the exhaustive enumeration. The results show that the algorithm converges to the optimal solution in early iterations with a reasonable CPU time.

\subsection{The Variable Capacity Case Versus the Fixed Capacity} Case. Table 5 shows the optimal plan for different market penetration rates assuming a fixed per-lane capacity. In this case, the capacity of each regular lane is set to 2400 vehicles per hour and the capacity of each AV lane is set to 4200 vehicles per hour, which equals to 1.75 $\left(\left(h_{\mathrm{AV}} / h_{\mathrm{HDV}}\right)=(1.5 / 0.85) \cong 1.75\right)$ times the per-lane capacity of a regular lane. The comparison of the fixed capacity with variable capacity cases indicates that not only the optimal plan varies at each market penetration rate but also the trend of estimated benefits obtained by the AV lane deployment is significantly overestimated. As shown in Table 5, the reduction in the total travel time caused by an AV lane deployment increased up to market penetration rate of $60 \%$ and remained constant with the increase of AV market size. Compared to its corresponding result in the variable capacity case, the benefits are overestimated in the fixed capacity case. The reason is that, in the variable capacity case, the base scenario total travel time $\left(\mathrm{TTT}_{\mathrm{bs}}\right)$ decreases as the AV market size grows. This decrease is due to the dependence of capacity to market penetration rate, while the fixed capacity case ignores the change of capacity and thus, takes the base scenario total travel time
TABle 5: The optimal plan for different market penetration rates (fixed capacity).

\begin{tabular}{|c|c|c|}
\hline $\begin{array}{l}\text { Market } \\
\text { penetration rate }\end{array}$ & Optimal plan & $\begin{array}{c}\text { Improvement in the } \\
\text { objective function (percent) }\end{array}$ \\
\hline 10 & 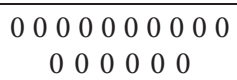 & 0.00 \\
\hline 20 & $\begin{array}{c}00110011010 \\
01100\end{array}$ & 0.39 \\
\hline 30 & $\begin{array}{c}10000011011 \\
00100\end{array}$ & 2.36 \\
\hline 40 & $\begin{array}{c}10100011111 \\
11101\end{array}$ & 5.99 \\
\hline 50 & 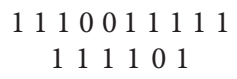 & 9.04 \\
\hline 60 & 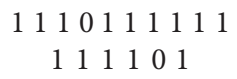 & 9.88 \\
\hline 70 & 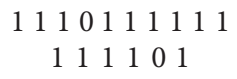 & 9.90 \\
\hline 80 & 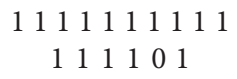 & 9.89 \\
\hline 90 & $\begin{array}{rrrrrrrrr}11 & 1 & 1 & 1 & 1 & 1 & 1 & 1 & 1 \\
1 & 1 & 1 & 1 & 0 & 1\end{array}$ & 9.91 \\
\hline
\end{tabular}

as constant. It can be concluded that the introduction of AVs to the network improved the $\mathrm{TTT}_{\mathrm{bs}}$ itself, as shown in Figure 5 and, therefore, the estimated benefit in the fixed capacity case is an overestimated value. This point can have significant implications in network planning and should be considered in the process of decision making by the network manager to have a reasonable expectation from the network improvement obtained by an AV lane deployment. 


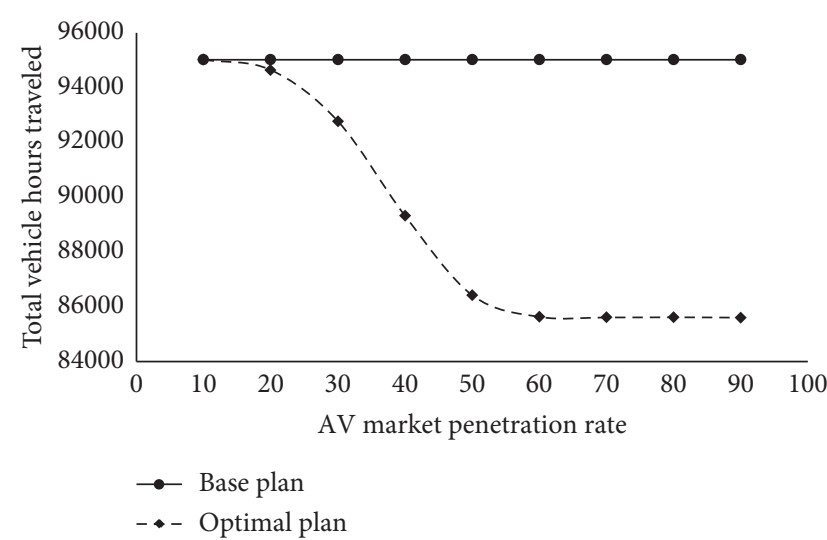

(a)

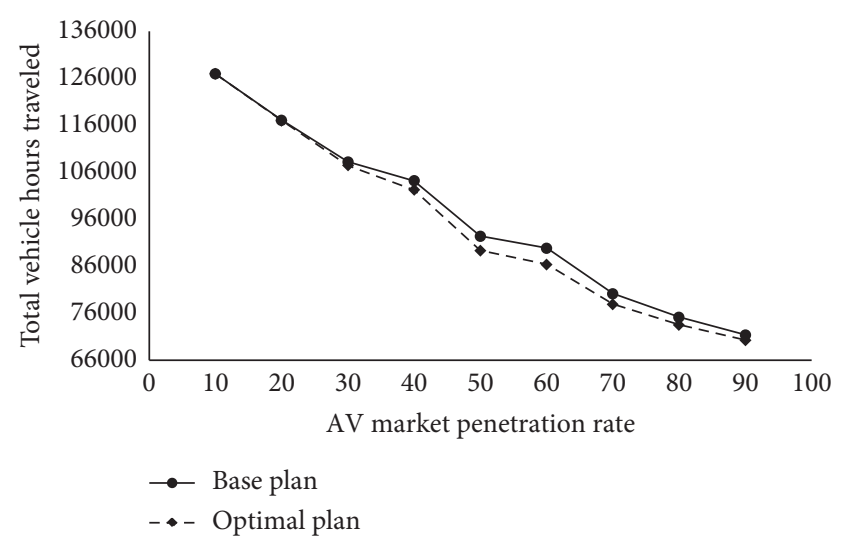

(b)

Figure 5: Total vehicle hours traveled in the network for different market penetration rates. (a) Variable capacity case. (b) Fixed capacity case.

\section{Conclusion}

This paper proposes a mathematical model that determines the best scheme of road space reallocation between AVs and HDV s by locating AV lanes in a transportation network. The bilevel optimization problem is formulated as a discrete network design problem which is an NP-hard problem and a hybrid machine-learning and optimization method is employed to solve it. The location of AV lanes is specified at the upper-level problem. The multiclass assignment problem at the lower level is formulated as a variational inequality since it is not a convex problem. The main contribution of the model is investigating the capacity impacts of autonomous vehicles at the network level. To achieve this goal, we used the BPR volume-delay function as the travel time function in the route choice procedure. The link capacity is one of the parameters of BPR function which is usually assumed as a constant value due to the link specifications. In this study the link capacity is considered as a function of AV proportion in a link, and, therefore, it can take different values according to the class-specific link flows.

The model was applied on the Sioux Falls network and the numerical results were presented at various market penetration rates. It was found that the benefits (i.e., reduction in total travel time) gained from AV lane deployment is highly affected by the market penetration rate. To derive benefits from the implementation of AV lanes requires relatively wide adaptation of AVs (i.e., more than $30 \%)$. The results indicate that the benefit is maximum at medium ranges (i.e., 60\%) of the market penetration rate. At low market penetration rates (i.e., $10 \%, 20 \%$, and $30 \%$ ), AV lane deployment does not lead to significant network improvements. The results also point out that ignoring the capacity variation would lead to different optimal plans as well as overestimation in benefits. A practical deployment plan during transition period, when market penetration rate grows from low levels to the saturation rate, is presented in this study. The practical plan considers the disturbances that adding or removing an AV lane may cause in the network.
This study can be followed up in different directions. In the proposed model, the total travel time serves as the objective function, while it is possible to add other social costs and account for different values of time of each user class. It is also interesting to examine the results using different capacity functions developed in various studies. The model can be extended to a multistage problem in which time is one of the dimensions. Since the introduction of AVs can affect travel demand and urban development, another field of research is to integrate the optimal AV lane location problem with AV travel impacts such as spatial distribution [44, 45], ridesharing [46], parking demand [47], and mode choice [48], which are examined separately in some previous studies.

\section{Data Availability}

The data used to support the findings of this study are available from Leblanc et al. [43].

\section{Conflicts of Interest}

The authors declare that they have no conflicts of interest.

\section{References}

[1] L. M. Clements and K. M. Kockelman, "Economic effects of automated vehicles," Transportation Research Record: Journal of the Transportation Research Board, vol. 2606, no. 1, pp. 106-114, 2017.

[2] D. J. Fagnant and K. Kockelman, "Preparing a nation for autonomous vehicles: opportunities, barriers and policy recommendations," Transportation Research Part A: Policy and Practice, vol. 77, pp. 167-181, 2015.

[3] T. Folsom, "Energy and autonomous urban land vehicles," IEEE Technology and Society Magazine, vol. 31, no. 2, pp. 28-38, 2012.

[4] W. Gruel and K. F. Stanford, "Assessing the long-term effects of autonomous vehicles: a speculative approach," Transportation Research Procedia, vol. 13, pp. 18-29, 2016.

[5] T. Litman, Vehicle Implementation Predictions, Victoria Transport Policy Institute, Victoria, Canada, 2018. 
[6] P. Bansal and K. M. Kockelman, "Forecasting Americans' long-term adoption of connected and autonomous vehicle technologies," Transportation Research Part A: Policy and Practice, vol. 95, pp. 49-63, 2017.

[7] L. J. Lavasani, E. K. Jin, and W. P. Du, "Market penetration model for autonomous vehicles on the basis of earlier technology adoption experience," Transportation Research, vol. 2597, no. 5, pp. 67-74, 2016.

[8] R. Shabanpour, A. Shamshiripour, and A. Mohammadian, "Modeling adoption timing of autonomous vehicles: innovation diffusion approach," Transportation, vol. 45, pp. 1607-1621, 2018.

[9] B. J. Cottam, "Transportation planning for connected autonomous vehicles: how it all fits together," Transportation Research Record: Journal of the Transportation Research Board, vol. 2672, no. 51, pp. 12-19, 2018.

[10] G.-L. Jia, R.-G. Ma, and Z.-H. Hu, "Review of urban transportation network design problems based on CiteSpace," Mathematical Problems in Engineering, vol. 2019, Article ID 5735702, 22 pages, 2019.

[11] B. Kuhn, G. Goodin, A. Ballard et al., "Managed lanes handbook," Texas Transportation Institute, College Station, TX, USA, 2005.

[12] A. Talebpour, H. S. Mahmassani, and A. Elfar, "Investigating the effects of reserved lanes for autonomous vehicles on congestion and travel time reliability," Transportation Research Record: Journal of the Transportation Research Board, vol. 2622, no. 1, pp. 1-12, 2017.

[13] L. J. Vander Laan and K. F. Sadabadi, "Operational performance of a congested corridor with lanes dedicated to autonomous vehicle traffic," International Journal of Transportation Science and Technology, vol. 6, no. 1, pp. 42-52, 2017.

[14] L. Ye and T. Yamamoto, "Impact of dedicated lanes for connected and autonomous vehicle on traffic flow throughput," Physica A: Statistical Mechanics and its Applications, vol. 512, pp. 588-597, 2018.

[15] A. Ghiasi, O. Hussain, Z. Qian, Sean), and X. Li, "A mixed traffic capacity analysis and lane management model for connected automated vehicles: a Markov chain method," Transportation Research Part B: Methodological, vol. 106, pp. 266-292, 2017.

[16] J. Ivanchev, A. Knoll, D. Zehe, S. Nair, and D. Eckhoff, "Potentials and implications of dedicated highway lanes for autonomous vehicles," 2017, https://arxiv.org/abs/1709. 07658.

[17] W. Wu, F. Zhang, W. Liu, and G. Lodewijks, "Modelling the traffic in a mixed network with autonomous-driving expressways and non-autonomous local streets," Transportation Research Part E: Logistics and Transportation Review, vol. 134, Article ID 101855, 2020.

[18] Z. Chen, F. He, L. Zhang, and Y. Yin, "Optimal deployment of autonomous vehicle lanes with endogenous market penetration," Transportation Research Part C: Emerging Technologies, vol. 72, pp. 143-156, 2016.

[19] D. A. Lazar, S. Coogan, and R. Pedarsani, "Capacity modeling and routing for traffic networks with mixed autonomy," in Proceedings of the 2017 IEEE 56th Annual Conference on Decision and Control (CDC), pp. 5678-5683, Melbourne, Australia, December 2017.

[20] E. Miandoabchi, F. Daneshzand, R. Zanjirani Farahani, and W. Y. Szeto, "Time-dependent discrete road network design with both tactical and strategic decisions," Journal of the Operational Research Society, vol. 66, no. 6, pp. 894-913, 2015.
[21] M. Bagherian, M. Mesbah, and L. Ferreira, "Using delay functions to evaluate transit priority at signals," Public Transport, vol. 7, no. 1, pp. 61-75, 2015.

[22] R. Z. Farahani, E. Miandoabchi, W. Y. Szeto, and H. Rashidi, "A review of urban transportation network design problems," European Journal of Operational Research, vol. 229, no. 2, pp. 281-302, 2013.

[23] M. J. Patriksson, The Traffic Assignment Problem: Models and Methods, Dover Publications, Mineola, NY, USA, 2015.

[24] Z. Chen, F. He, Y. Yin, and Y. Du, "Optimal design of autonomous vehicle zones in transportation networks," Transportation Research Part B: Methodological, vol. 99, pp. 44-61, 2017.

[25] S. C. Dafermos, "The traffic assignment problem for multiclass-user transportation networks," Transportation Science, vol. 6, no. 1, pp. 73-87, 1972.

[26] P. Marcotte and L. Wynter, "A new look at the multiclass network equilibrium problem," Transportation Science, vol. 38, no. 3, pp. 282-292, 2004.

[27] A. T. Mehr and R. Horowitz, "How will the presence of autonomous vehicles affect the equilibrium state of traffic networks?,” 2019, https://arxiv.org/abs/1901.05168.

[28] M. W. Levin and S. D. Boyles, "Effects of autonomous vehicle ownership on trip, mode, and route choice," Transportation Research Record: Journal of the Transportation Research Board, vol. 2493, no. 1, pp. 29-38, 2015.

[29] J. Wang, S. Peeta, and X. He, "Multiclass traffic assignment model for mixed traffic flow of human-driven vehicles and connected and autonomous vehicles," Transportation Research Part B: Methodological, vol. 126, pp. 139-168, 2019.

[30] P. Tientrakool, Y. Ho, and N. F. Maxemchuk, "Highway capacity benefits from using vehicle-to-vehicle communication and sensors for collision avoidance," in Proceedings of the 2011 IEEE Vehicular Technology Conference (VTC Fall), pp. 1-12, San Francisco, CA, USA, September 2011.

[31] D. Chen, S. Ahn, M. Chitturi, and D. A. Noyce, "Towards vehicle automation: roadway capacity formulation for traffic mixed with regular and automated vehicles," Transportation Research Part B: Methodological, vol. 100, pp. 196-221, 2017.

[32] M. W. Lazar, S. D. Chandrasekher, R. Pedarsani, and D. Sadigh, "Maximizing road capacity using cars that influence people," 2018, https://arxiv.org/abs/1807.04414.

[33] S. Dafermos, "Traffic equilibrium and variational inequalities," Transportation Science, vol. 14, no. 1, pp. 42-54, 1980.

[34] M. J. Smith, "The existence, uniqueness and stability of traffic equilibria," Transportation Research Part B: Methodological, vol. 13, no. 4, pp. 295-304, 1979.

[35] O. Ben-Ayed, D. E. Boyce, and C. E. Blair, “A general bilevel linear programming formulation of the network design problem," Transportation Research Part B: Methodological, vol. 22, no. 4, pp. 311-318, 1988.

[36] A. Ghaffari, M. Mesbah, and A. Khodaii, "Designing a transit priority network under variable demand," Transportation Letters, pp. 1-14, 2019.

[37] M. Mesbah, R. Thompson, and S. Moridpour, "Bilevel optimization approach to design of network of bike lanes," Transportation Research Record: Journal of the Transportation Research Board, vol. 2284, no. 1, pp. 21-28, 2012.

[38] L. J. Leblanc, "An algorithm for the discrete network design problem," Transportation Science, vol. 9, no. 3, pp. 183-199, 1975.

[39] H. Poorzahedy and H. S. Rouhani, "Hybrid meta-heuristic algorithms for solving network design problem," European 
Journal of Operational Research, vol. 182, no. 2, pp. 578-596, 2007.

[40] S. Shiripour, N. Mahdavi-Amiri, and I. Mahdavi, "A transportation network model with intelligent probabilistic travel times and two hybrid algorithms," Transportation Letters, vol. 9, no. 2, pp. 90-122, 2017.

[41] T. Zhang, G. Ren, and Y. Yang, "Transit route network design for low-mobility individuals using a hybrid metaheuristic approach," Journal of Advanced Transportation, vol. 2020, Article ID 7059584, 12 pages, 2020.

[42] S. A. Bagloee, M. Asadi, M. Sarvi, and M. Patriksson, "A hybrid machine-learning and optimization method to solve bi-level problems," Expert Systems with Applications, vol. 95, pp. 142-152, 2018.

[43] L. J. LeBlanc, E. K. Morlok, and W. P. Pierskalla, "An efficient approach to solving the road network equilibrium traffic assignment problem," Transportation Research, vol. 9, no. 5, pp. 309-318, 1975.

[44] S. Carrese, M. Nigro, S. M. Patella, and E. Toniolo, "A preliminary study of the potential impact of autonomous vehicles on residential location in Rome," Research in Transportation Economics, vol. 75, pp. 55-61, 2019.

[45] J. Meyer, O. M. Becker, P. M. Bösch, and K. W. Axhausen, "Autonomous vehicles: the next jump in accessibilities?" Research in Transportation Economics, vol. 62, no. 2, pp. 80-91, 2017.

[46] A. T. Moreno, A. Michalski, C. Llorca, and R. Moeckel, "Shared autonomous vehicles effect on vehicle-Km traveled and average trip duration," Journal of Advanced Transportation, vol. 2018, Article ID 8969353, 10 pages, 2018.

[47] W. Zhang and S. Guhathakurta, "Parking spaces in the age of shared autonomous vehicles: how much parking will we need and where?" Transportation Research Record: Journal of the Transportation Research Board, vol. 2651, no. 1, pp. 80-91, 2017.

[48] P. Ashkrof, G. Homem de Almeida Correia, O. Cats, and B. van Arem, "Impact of automated vehicles on travel mode preference for different trip purposes and distances," Transportation Research Record: Journal of the Transportation Research Board, vol. 2673, no. 5, pp. 607-616, 2019. 